\title{
Exchange Coupled NiFe/NiMn Bilayer Studied by a Vector Network Analyzer Ferromagnetic Resonance
}

\author{
H. GŁowiński ${ }^{a}$, K. ZaŁȩSKi ${ }^{a}$, J. Sprada ${ }^{b}$ And J. Dubowik ${ }^{a, *}$ \\ ${ }^{a}$ Institute of Molecular Physics, PAS, M. Smoluchowskiego 17, 60-179 Poznań, Poland \\ ${ }^{b}$ Department of Physics, A. Mickiewicz University, Umultowska 85, 61-614 Poznań, Poland
}

\begin{abstract}
Using cavity ferromagnetic resonance and vector network analyzer ferromagnetic resonance unidirectional, uniaxial and rotatable anisotropies were determined in permalloy $(30 \mathrm{~nm}) / \mathrm{NiMn}(56 \mathrm{~nm})$. Resonance field dependence on in-plane angle clearly shows unidirectional and uniaxial anisotropy. Rotatable anisotropy, which acts as internal magnetic field independent of a direction of external magnetic field, is observed on vector network analyzer ferromagnetic resonance as upward shift of the resonance frequency of $\mathrm{Py} / \mathrm{NiMn}$ compared to Py film. Even for a small exchange bias field, this shift is significantly large.
\end{abstract}

PACS: 75.70.-i, 75.30.Et, 75.30.Gw, 75.70.Cn, 76.50.+g

\section{Introduction}

Well known phenomenon of exchange bias (EB), discovered more than fifty years ago [1], is still actively investigated for its importance for spintronic devices as well as for deeper understanding of fragile interactions at ferromagnetic/antiferromagnetic (FM/AFM) interfaces [2]. Films with FM/AFM interfaces may be also attractive for microwave devices, because of the observed upward frequency shift $[3,4]$. In the second aspect, some of important issues concerning magnetization dynamics of an FM layer exchange coupled to AFM are not fully understood [5] including rotatable anisotropy that describes an enhancement of the coercive field for magnetization reversal measurements and a negative line shift in ferromagnetic resonance (FMR) measurements [6].

The aim of the present contribution is to show that even for weak EB effect (shift of hysteresis loop) the rotatable anisotropy is substantial in permalloy/NiMn bilayers. For this purpose we have employed novel technique of broadband FMR with vector network analyzer (VNA-FMR).

\section{Experimental and results}

Thin film permalloy/NiMn (Py/NiMn) structures were prepared by magnetron sputtering with base pressure better than $2 \times 10^{-7}$ Torr and Ar pressure 8 mTorr. The films: $\mathrm{Py}(30 \mathrm{~nm})$ and $\mathrm{Py}(30 \mathrm{~nm}) / \operatorname{NiMn}(56 \mathrm{~nm})$

\footnotetext{
* corresponding author; e-mail: dubowik@ifmpan.poznan.pl
}

were deposited on single crystalline $\mathrm{Si}(100)$ substrates. The $\operatorname{Py}(30 \mathrm{~nm}) / \mathrm{NiMn}(56 \mathrm{~nm})$ bilayer was annealed in magnetic field of 800 Oe at temperature $T_{\mathrm{a}}$ of $300^{\circ} \mathrm{C}$ in $10^{-6}$ Torr for several hours. Hysteresis loop was acquired by vibrating sample magnetometer (VSM). Dynamic measurements of the magnetization were carried out by standard FMR X-band spectrometer and VNA-FMR.

The frequency domain measurements were performed using a coplanar waveguide. It has a $50 \Omega$ characteristic impedance to match the impedance of VNA. The sample was placed upside down on top of coplanar waveguide with the film on a center conductor. The direction of cooling field, which is easy direction, was parallel to external dc magnetic. The frequency was swept up to $15 \mathrm{GHz}$. The resonance frequency was obtained from Lorentzian fit to $\left|S_{21}\right|$ component of scattering matrix [7].

Figure 1 shows the resonance field dependence on in-plane angle between external magnetic field and anisotropy direction at $9.18 \mathrm{GHz}$. It is seen that the Py/NiMn bilayer exhibits both unidirectional $\left(H_{\mathrm{eb}}\right)$ and uniaxial $\left(H_{\mathrm{u}}\right)$ anisotropy. The difference between resonance field at two local minima is twice the EB field $\left(H_{\mathrm{eb}}\right)$, which corresponds to a field-shift of hysteresis loop.

Fit to experimental data yields the values of $H_{\mathrm{eb}}$ and anisotropy field $H_{\mathrm{u}}$. Continuous line represents a fit to the experimental data using the standard resonance condition with the total free energy comprising magnetostatic shape anisotropy energy, uniaxial anisotropy energy in-plane, Zeeman energy, unidirectional exchange energy, and the rotatable anisotropy energy [6]. Only 


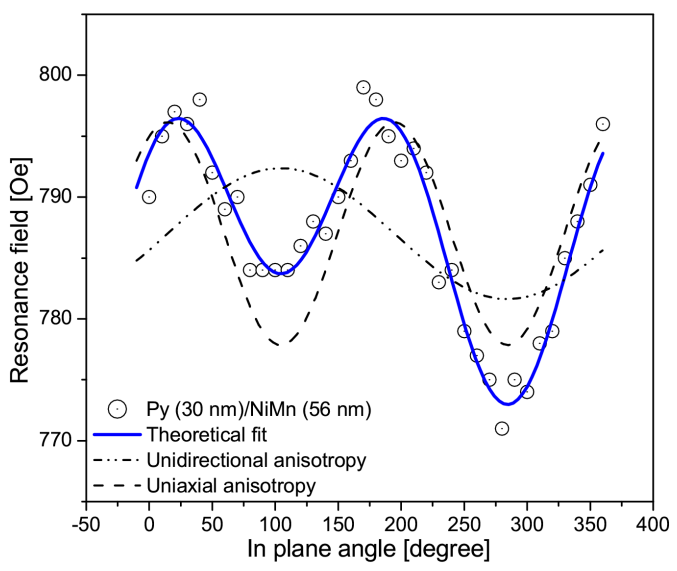

Fig. 1. In-plane angular distribution of the resonance field in permalloy $(30 \mathrm{~nm}) / \mathrm{NiMn}(56 \mathrm{~nm})$ measured with FMR. The dots are experimental points and the curve is a fit.

$H_{\mathrm{eb}}=5.4$ Oe and $H_{\mathrm{u}}=9.1$ Oe can be undoubtedly extracted from fitting procedure. Rotatable anisotropy $H_{\text {ra }}$ cannot be obtained because it is related to magnetization $M$.

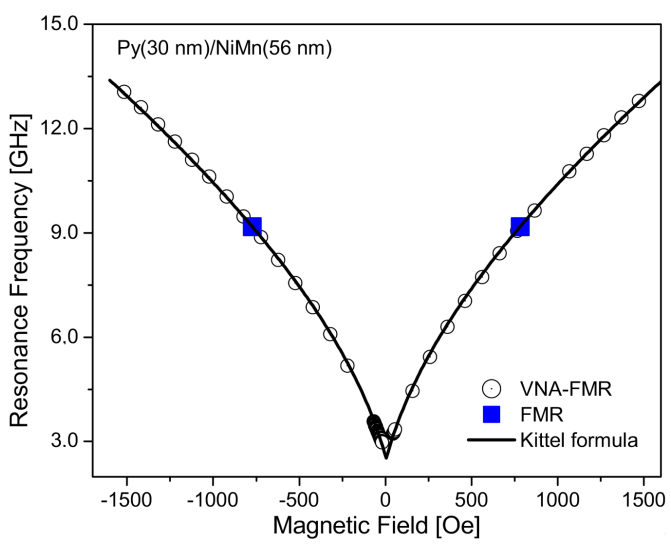

Fig. 2. VNA-FMR measurement of $\mathrm{Py}(30 \mathrm{~nm}) /$ $\operatorname{NiMn}(56 \mathrm{~nm})$. The dots represent experimental points and line is a fit with Kittel formula. The square, blue marks are FMR points at angles, which correspond to VNA-FMR directions of measurement.

Figure 2 shows the resonance frequency as a function of the external magnetic field investigated with VNA-FMR. The experimental data was fitted with the Kittel formula $[3,8]$.

$$
\begin{aligned}
f_{\mathrm{r}} & =\frac{\gamma}{2 \pi}\left[\left(H_{\mathrm{u}}+H_{\mathrm{ra}}+\left|H-H_{\mathrm{eb}}\right|\right)\right. \\
& \left.\times\left(H_{\mathrm{u}}+H_{\mathrm{ra}}+\left|H-H_{\mathrm{eb}}\right|+4 M \pi\right)\right]^{1 / 2} .
\end{aligned}
$$

Fitting procedure yields the following parameters: $H_{\mathrm{u}}+H_{\mathrm{ra}}, H_{\mathrm{eb}}$ and $M$. To calculate $H_{\mathrm{ra}}$, value of $H_{\mathrm{u}}$ was taken from FMR in-plane measurement and set constant during fitting procedure. All values of the extracted parameters are listed in Table. Both the magnetization $M$ and the uniaxial anisotropy $H_{\mathrm{u}}$, which describe Py layer, are similar for both samples. The unidirectional and rotatable anisotropy describe NiMn layer and the FM/AFM interface. In Fig. 2 there are also shown two points from FMR for comparison. Values of FMR resonance field are taken for angles which correspond to direction of exchange bias field and antiparallel to it.

Comparison of magnetic properties.

TABLE

\begin{tabular}{l|c|c|c|c}
\hline \hline \multirow{2}{*}{$\begin{array}{c}\text { Sample } \\
{[\mathrm{nm}]}\end{array}$} & $M$ & $H_{\mathrm{u}}$ & $H_{\mathrm{ra}}$ & $H_{\mathrm{eb}}$ \\
\cline { 2 - 5 } & \multicolumn{4}{|c}{$[\mathrm{Oe}]$} \\
\hline $\mathrm{Py}(30)$ & 792.3 & 7.2 & 0 & 0 \\
$\mathrm{Py}(30) / \operatorname{NiMn}(56)$ & 800.8 & 9.1 & 58.3 & 4.4
\end{tabular}

In Fig. 3 Py/NiMn sample is compared with a Py film with magnetic parameters listed in Table. Exchange coupled film is shifted to higher frequency due to rotatable anisotropy. It acts as internal field, which adds to external dc field. It is independent from in-plane angle.

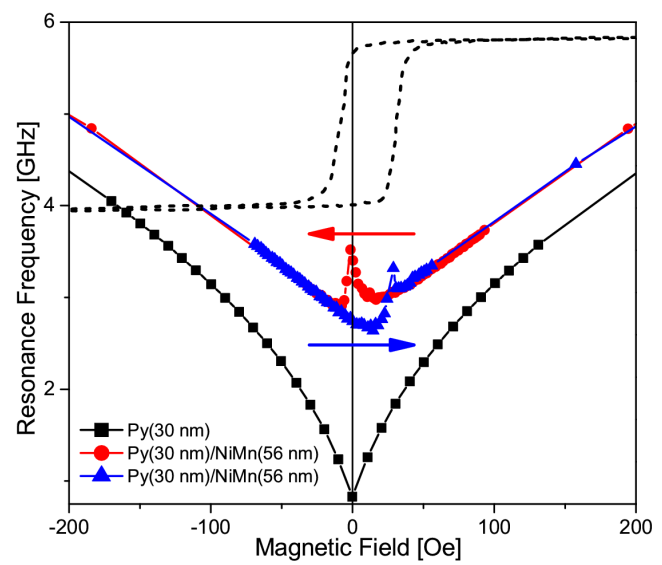

Fig. 3. Low magnetic field region. VNA-FMR in $\mathrm{Py} / \mathrm{NiMn}$ (red circles and blue triangles for two directions of changing the external field) compared with VNA-FMR in Py (black squares). Hysteresis loop (dashed line) of $\mathrm{Py} / \mathrm{NiMn}$ bilayer is inserted for comparison.

Changing external magnetic field from positive to negative results in different experimental data for $\mathrm{Py} / \mathrm{NiMn}$ than changing the field in the opposite way. Figure 3 shows the dispersion relation in more detail in the low field region. Hysteresis of the resonance frequency as a function of the external magnetic field seen in this region is due to reorganization of FM/AFM interface. Therefore Bilzer et al. [8] suggest that $H_{\mathrm{eb}}$ field changes during magnetization reversal of FM film. For comparison, a hysteresis loop is inserted in Fig. 3. It is seen that this ambiguity in dispersion relation occurs in field region limited with coercive fields.

Resonance frequency dependence on the external field has two local maxima in vicinity of the fields corresponding to magnetization reversals. In this region the Py mag- 
netization rotates and experiences metastable behavior. The Lorentzian peak in VNA-FMR broadens largely and therefore the fit is not perfect.

From the above discussion and from Table one can see that there is a significant upward frequency shift. Probably AFM layer consists of grains, which are coupled to FM layer. Some of these grains are pinned and have influence on $H_{\mathrm{eb}}$ field. The rest of them can rotate during reversal of magnetization and induces the rotatable anisotropy. These rotating grains cannot respond to microwave magnetic field of high frequency. Their anisotropy direction takes the same direction as the external dc magnetic film, but are rigid for the microwave field. Therefore the AFM grains act as microscopic source of anisotropy which has an easy direction always parallel to magnetization direction.

\section{Conclusions}

Magnetic parameters (uniaxial anisotropy $H_{\mathrm{u}}$, unidirectional exchange $H_{\mathrm{eb}}$ and the rotatable anisotropy $H_{\mathrm{ra}}$ ) were determined using VNA-FMR and FMR. Even for small $H_{\mathrm{eb}}$ field (it correspond to field-shift of hysteresis loop) there is a significant rotatable anisotropy $\left(H_{\text {ra }}\right)$. It is responsible for an upward frequency shift independent of the in-plane angle. It originates from reorganization of magnetic moments of AFM grains at interface.

\section{References}

[1] W.H. Meiklejohn, C.P. Bean, Phys. Rev. 102, 1413 (1956).

[2] J. Nogues, I.K. Schuller, J. Magn. Magn. Mater. 192, 203 (1999).

[3] B.K. Kuanr, S. Maat, S. Chandrashekariaih, V. Veerakumar, R.E. Camley, Z. Celinski, J. Appl. Phys. 103, 07C107 (2008)

[4] J. Chen, D. Tang, Y. Li, B. Zhang, Y. Yang, M. Lu, H. Lu, J. Magn. Magn. Mater. 322, 3109 (2010).

[5] M. Gloanec, S. Rioual, B. Lescop, R. Zuberek, R. Szymczak, P. Aleshkevych, B. Rouvellou, Phys. Rev. B 82, 144433 (2010).

[6] R.D. McMichael, M.D. Stiles, P.J. Chen, W.F. Egelhoff, Jr., Phys. Rev. B 58, 8605 (1998).

[7] B.K. Kuanr, R.E. Camley, Z. Celinski, J. Magn. Magn. Mater. 286, 276 (2005).

[8] C. Bilzer, T. Devolder, Joo-Von Kim, C. Chappert, M. Ruehrig, L. Baer, J. Appl. Phys. 106, 063918 (2009). 